Check for updates

Cite this: J. Mater. Chem. C, 2021, 9, 5771

Received 30th September 2020, Accepted 20th March 2021

DOI: $10.1039 / d 0 t c 04667 a$

rsc.li/materials-c

\section{Ligand-assisted solid phase synthesis of mixed-halide perovskite nanocrystals for color-pure and efficient electroluminescence $\dagger$}

\author{
Simon F. Solari, (D) ${ }^{a}$ Sudhir Kumar, (D) a Jakub Jagielski, (D) ${ }^{a}$ Nikolas M. Kubo, ${ }^{a}$ \\ Frank Krumeich (D) ${ }^{b}$ and Chih-Jen Shih (D) *a
}

\begin{abstract}
Colloidal nanocrystals (NCs) of lead halide perovskites have generated considerable interest in the fabrication of optoelectronic devices, such as light emitting-diodes (LEDs), because of their tunable optical bandgap, narrow spectral width, and high defect tolerance. However, the inhomogeneous halide distribution within individual NCs remains a critical challenge in order to obtain color-stable electroluminescence in mixed-halide systems. Here, we demonstrate a new post-synthetic approach, ligand-assisted solid phase synthesis (LASPS), for the preparation of electroluminescent colloidal NCs of methylammonium (MA) lead halide perovskites, at room temperature. The slow reaction kinetics preserves the morphology, size, and shape in the resulting NCs whose emission covers the entire visible spectral region with photoluminescence $(\mathrm{PL})$ quantum yields (QYs) of up to $>90 \%$ and colloidal stability up to several months. The LEDs fabricated using the prepared mixed-halide NCs display narrowband electroluminescence (EL) ranging from 476 to $720 \mathrm{~nm}$. The optimized red LEDs exhibit an external quantum efficiency, $\eta_{\text {ext, }}$ of up to $2.65 \%$, with the CIE 1931 color coordinates of $(0.705,0.290)$, nearly identical to those of the red primary in the recommendation (rec.) 2020 standard $(0.708,0.292)$.
\end{abstract}

Colloidal nanocrystals (NCs) of organic-inorganic hybrid perovskites (OIHPs) have emerged as the most promising class of semiconductors for optoelectronic applications, including solar cells, lasers, and light-emitting diodes (LEDs). ${ }^{1-5}$ OIHPs have the general formula of $\mathrm{ABX}_{3}$, where $\mathrm{A}$ - and B-sites are cationic species and $\mathrm{X}$ represents halides. ${ }^{6}$ The emerging semiconductor system has demonstrated a wide range of tunability in optical and photophysical characteristics through composition engineering owing to its soft nature, which also leads to fast migration of anionic species and high defect tolerance. ${ }^{7}$ In addition to reduced dimensionality, ${ }^{8}$ the composition engineering, including halide and A- and B-site mixing, has shown its versatility to tune the optical bandgap of perovskite NCs. ${ }^{9-12}$ Indeed, although the quantum-confined OIHP NCs exhibit a high exciton binding energy without sacrificing the photoluminescence (PL) quantum yields (QYs), the large surface-to-volume ratio demands a large amount of capping ligands, ${ }^{13}$ which compromise the charge transport characteristics that are essential for obtaining high-efficiency

\footnotetext{
${ }^{a}$ Institute for Chemical and Bioengineering, Department of Chemistry and Applied Biosciences, ETH Zürich, Vladimir Prelog Weg 1, CH-8093 Zürich, Switzerland. E-mail: chih-jen.shih@chem.ethz.ch

${ }^{b}$ Laboratory of Inorganic Chemistry, Department of Chemistry and Applied Biosciences, ETH Zürich, Vladimir Prelog Weg 1, CH-8093 Zürich, Switzerland

$\dagger$ Electronic supplementary information (ESI) available. See DOI: 10.1039/d0tc04667a
}

electroluminescence (EL). ${ }^{14}$ In contrast, the three-dimensional (3D) OIHP NCs can be stabilized with a relatively small amount of ligands, ${ }^{15}$ and the emission wavelength can be readily tuned by composition engineering. ${ }^{9}$ Accordingly, we consider them to be better candidates for electroluminescent materials.

As far as halide mixing is concerned, there are two general approaches: (i) direct synthesis of mixed-halide perovskites via mixing different halide precursors, ${ }^{16}$ and (ii) post-synthetic anion exchange of OIHP and pure inorganic lead halide perovskites. ${ }^{17-21}$ The latter is analogous to the well-established surface modification strategies, such as ligand exchange, in colloidal quantum dot synthesis for enhancing stability and optoelectronic performance. ${ }^{20,22-27}$ Indeed, as the halide molecular orbitals strongly contribute to the valence band maximum (VBM) of the resulting perovskite compound, a variation in the halide composition would regulate the VBM and the optical bandgap. ${ }^{28}$ The first post-synthetic anion exchange in inorganic $\mathrm{CsPbX}_{3}$ was reported by Nedelcu et al., ${ }^{17}$ by injecting a halide precursor solution at elevated temperatures. Similar strategy was thereafter used to synthesize mixed-halide perovskite NCs. ${ }^{9}$ Other approaches are also reported. For example, Parobek et al. demonstrated a photo-induced anion exchange of colloidal $\mathrm{CsPbBr}_{3} \mathrm{NCs}$ in dihalomethane solvents; the wavelength and intensity of photoexcitation source could control the degree of halide mixing. However, only $\mathrm{Br} / \mathrm{Cl}$ mixing could work. ${ }^{29}$ Yoon et al. reported a 
reversible post-synthetic anion exchange route using the haloalkane solvents with a strong nucleophile trioctylphosphine (TOP). ${ }^{30}$ The nucleophile TOP promotes halogen-carbon bond dissociation through the $\mathrm{S}_{\mathrm{N}} 2$ reaction. Guhrenz et al. also reported a solid state post-synthetic anion exchange reaction by mixing solid potassium halides ( $\mathrm{KX}$, where $\mathrm{X}=\mathrm{Cl}, \mathrm{Br}$, or I) in an NC colloidal solution, followed by evaporating the solvents, ${ }^{31}$ but the reaction kinetics was considerably slow. Accordingly, the abovementioned methods often require complex synthetic protocols at elevated temperatures, which may hinder large-scale synthesis.

Here, we present a facile post-synthetic anion exchange method, ligand-assisted solid phase synthesis (LASPS), for the preparation of highly luminescent methylammonium lead halide $\left(\mathrm{MAPbX}_{3}\right)$ NCs. The proposed synthetic protocol is entirely performed at room temperature and under ambient conditions. The synthesized NCs were directly used in light-emitting diode (LED) devices. We demonstrate their maximum external quantum efficiencies, $\eta_{\text {ext }}$, ranging from $0.4 \%$ (476 nm) to $2.65 \%$ (656 nm), as compared to $3.5 \%$ based on parent $\mathrm{MAPbBr}_{3} \mathrm{NCs}(524 \mathrm{~nm})$. Finally, we further demonstrate an electric-field driven irreversible post-synthetic anion exchange technique for the OIHP NCs.

We synthesized the parent $\mathrm{MAPbBr}_{3}$ colloidal NCs dispersed in toluene using the ligand-assisted reprecipitation (LARP) method, followed by mixing with a solid powder of halide precursor and a small amount of ligands in a flask at room temperature (Fig. 1a; for details, see ESI $\dagger$ ). Methylammonium halide (MAX, $\mathrm{X}=\mathrm{Cl}$ or I) powder was used as the solid source to ensure that only anion exchange takes place. Two surfactants, oleylamine (OAM) and oleic acid (OLA), were used, identical to those in the parent $\mathrm{MAPbBr}_{3} \mathrm{NC}$ preparation. Upon mixing the reactants, the solution color gradually turned either dark or colorless depending on the halide precursors used (ESI, $\dagger$ Fig. S1). Fig. 1b presents a schematic diagram describing the halide exchange process. Specifically, upon mixing of halide powder and surfactants, halide ions get gradually dissolved in the nonpolar toluene by forming reverse micelles. ${ }^{32}$ The formation of halide-containing micelles increases the chemical potential of the halide ions, thereby slowly replacing the bromide ions in the perovskite NC lattices. The protocol was successful in both $\operatorname{MAPb}(\mathrm{Br} / \mathrm{I})_{3}$ and $\mathrm{MAPb}(\mathrm{Br} / \mathrm{Cl})_{3}$ systems. Prior to device fabrication, the excess ligands and unreacted species in solution were removed by washing with anti-solvents. A number of anti-solvents, including methyl acetate (MeOAc), ethyl acetate (EtOAc), dimethyl sulfoxide (DMSO), propionitrile, acetonitrile (ACN), acetone, $n$-butanol, chloroform, and their mixtures, have been investigated to maximize the perovskite NC concentration. As reported in the literature, the anti-solvent washing often considerably compromises the yield. ${ }^{33}$ After extensive experimentation, we found that MeOAc is the most optimal antisolvent for the purification of parent $\mathrm{MAPbBr}_{3}$ and $\mathrm{MAPb}(\mathrm{Br} /$ $\mathrm{Cl})_{3}$ NCs, and both MeOAc and $n$-butanol are highly effective anti-solvents for $\mathrm{MAPb}(\mathrm{Br} / \mathrm{I})_{3}$ NCs.

After halide exchange, the majority of parent bromide ions were substituted by either chloride or iodide ions, as revealed by the energy dispersive X-ray spectroscopy (EDXS) (ESI,† Fig. S2-S8). We find that the long-chain hydrocarbon ligands OLA and OAM play a crucial role in the reaction system by partially dissolving the MAX salts, such that excess halides become accessible for anion exchange. The OAM ligands are protonated by OLA to yield positively charged ammonium, so that they can form reverse micelles by surrounding the halide ions. We notice that the halide exchange kinetics is relatively slow ranging from few minutes to several hours, hypothetically due to the low solubility of halide ions in toluene. More experiments were carried out to estimate the proper amount of halide powder used in LASPS (ESI, $\dagger$ Fig. S9 and S10).

Taking $\operatorname{MAPb}(\mathrm{Br} / \mathrm{I})_{3}$ NCs as an example, we notice that the reaction reaches equilibrium within 1.5 hours; the emission

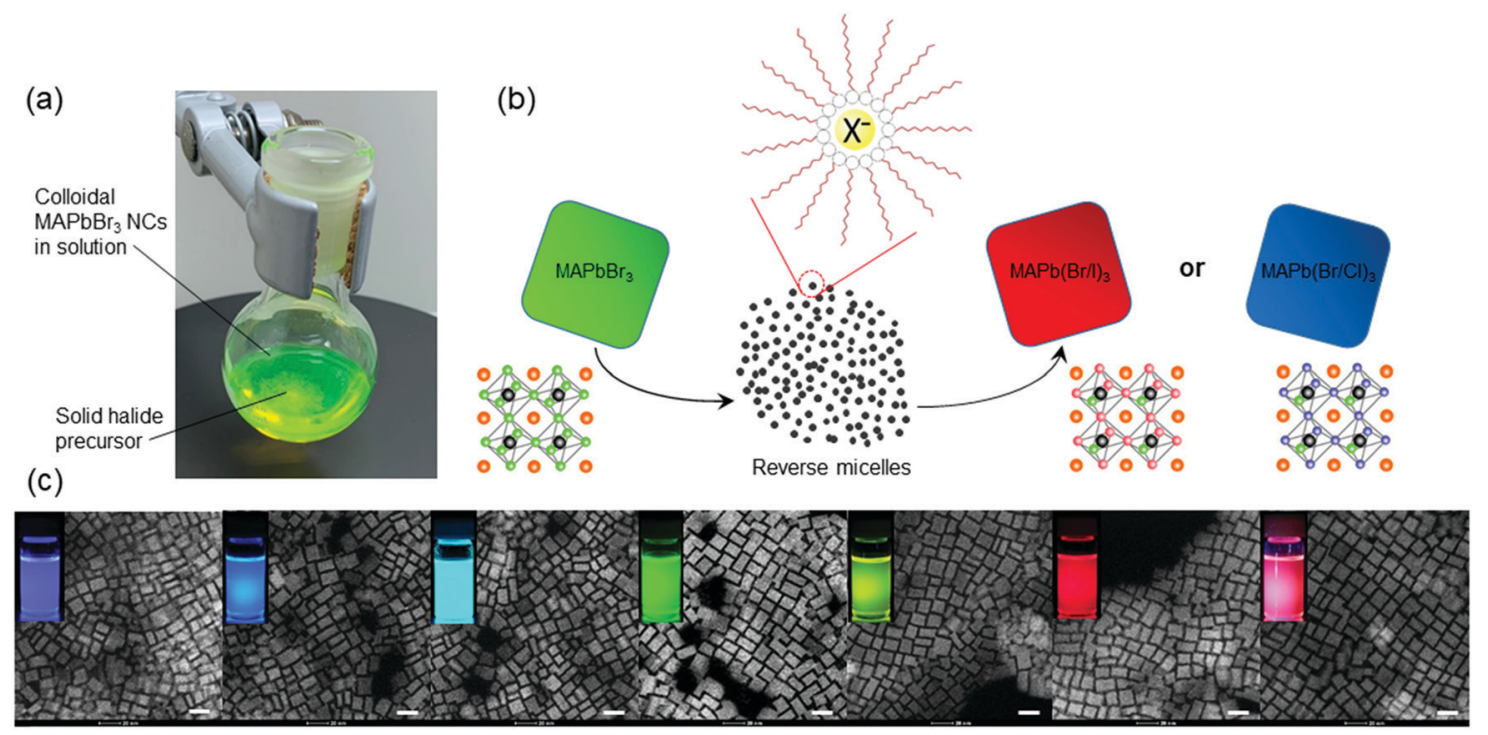

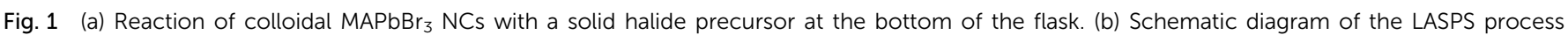

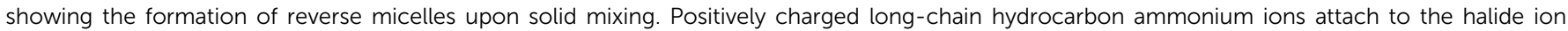

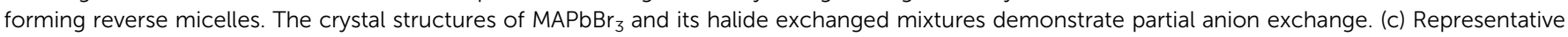
transmission electron micrographs and photographs under UV excitation for the synthesized MAPbX 3 NC solutions (scale bar: 20 nm). 
wavelengths between 1.5 and 17 hours did not show any noticeable difference (ESI, $\dagger$ Fig. S11). Mixed-halide NCs can be obtained by either terminating the reaction before reaching equilibrium or by mixing fully exchanged halide NCs with the parent $\mathrm{MAPbBr}_{3}$ NCs via the internanocrystal exchange (ESI, $\dagger$ Table S1). We also noticed that anionic mixing between iodide and chloride did not occur because of a considerable difference between their ionic radii (220 and $181 \mathrm{pm}$, respectively). ${ }^{34}$ Luminescent mixed-halide OIHP NCs covering the entire visible spectrum were synthesized (Fig. 1c). Their transmission electron micrographs suggest that the NC morphology, size, and shape are preserved upon halide mixing, consistent with the directly synthesized $\mathrm{MAPbX}_{3}$ NCs. $^{35,36}$

It is well-known that at room temperature, the crystalline phase of pure $\mathrm{MAPbCl}_{3}$ and $\mathrm{MAPbBr}_{3}$ is cubic but transforms to tetragonal in $\mathrm{MAPbI}_{3} \cdot{ }^{35-39}$ The cubic phase in $\mathrm{MAPbI}_{3}$ can only become thermodynamically stable at temperatures greater than $330 \mathrm{~K}^{40,41}$ Fig. 2 compares the X-ray diffraction (XRD) patterns of parent $\mathrm{MAPbBr}_{3}$ and the mixed-halide $\mathrm{MAPb}(\mathrm{Br} / \mathrm{Cl})_{3}$ and $\mathrm{MAPb}(\mathrm{Br} / \mathrm{I})_{3} \mathrm{NC}$ systems considered here. The parent $\mathrm{MAPbBr}_{3}$ is confirmed to be of the cubic phase, as revealed by the measured $d$-spacings of 5.82 and $4.12 \AA$ for the (100) and (110) planes, respectively, consistent with the literature. ${ }^{38,39}$ Interestingly, the crystallographic features of $\mathrm{MAPbBr}_{3}$ and $\mathrm{MAPb}(\mathrm{Br} / \mathrm{Cl})_{3}$ NCs are nearly identical. The lattice expansion and contraction upon iodide and chloride alloying are revealed by the degree of peak shifting. The $d$-spacing corresponding to the (100) peak in the cubic phase for fully reacted $\mathrm{MAPb}(\mathrm{Br} / \mathrm{Cl})_{3}$ perovskites is $5.63 \AA$, which is similar to those reported for cubic $\mathrm{MAPb}(\mathrm{Br} / \mathrm{Cl})_{3}$ alloys. ${ }^{42}$ Upon exchanging $\mathrm{Br}$ with $\mathrm{I}$, the X-ray diffraction peaks are gradually shifted towards lower angles due to lattice expansion. We observe splitting of the (100) plane peak to $2 \theta$ of $14.39^{\circ}$ and $14.82^{\circ}$ in the fully exchanged $\mathrm{MAPb}(\mathrm{Br} / \mathrm{I})_{3}$ system, which could be attributed to a transition between the cubic and tetragonal phase. Note that the pure $\mathrm{MAPbBr}_{3}$ has a cubic crystal structure, whereas pure $\mathrm{MAPbI}_{3}$ has a tetragonal crystal structure at room temperature. $^{39}$ It has been reported that when $x>0.2$ in the $\operatorname{MAPb}\left(\mathrm{Br}_{x} \mathrm{I}_{1-x}\right)_{3} \mathrm{NCs}$, the phase transition from tetragonal to a cubic would take place. ${ }^{43}$ Accordingly, we deduce that the I-rich

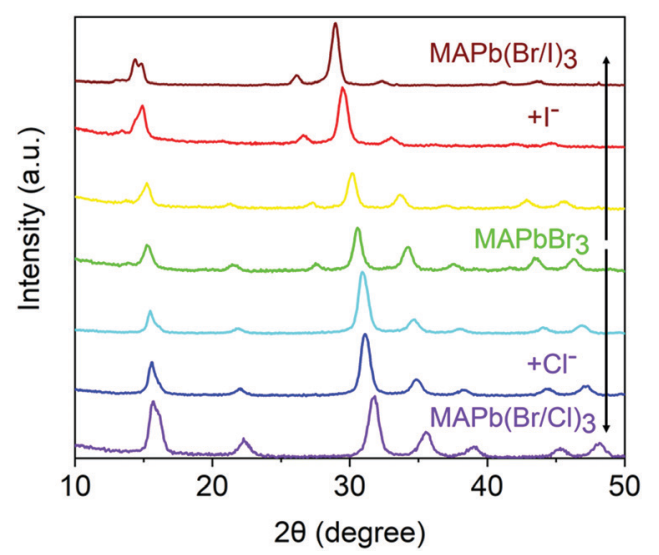

Fig. 2 Powder X-ray diffraction (XRD) patterns of the synthesized MAPbX 3 films.
$\operatorname{MAPb}(\mathrm{Br} / \mathrm{I})_{3}$ NCs are composed of both crystal phases, which is completely different from the parent $\mathrm{MAPbBr}_{3} \mathrm{NCs}$.

The absorption and photoluminescence (PL) spectra of parent $\mathrm{MAPbBr}_{3}$ and mixed-halide NC solutions are shown in Fig. $3 \mathrm{a}$ and $\mathrm{b}$, and ESI, $\dagger$ Fig. S12. Their PL quantum yields, $\eta_{\mathrm{PL}}$, are also shown in Fig. 3a. All samples show narrowband emission with the peak positions, $\lambda_{\mathrm{PL}}$, covering the entire visible spectral region. The parent $\mathrm{MAPbBr}_{3}$ and the fully reacted $\mathrm{MAPb}(\mathrm{Br} / \mathrm{I})_{3}$ and $\operatorname{MAPb}(\mathrm{Br} / \mathrm{Cl})_{3}$ NCs exhibit $\eta_{\mathrm{PL}}$ values of $91 \%\left(\lambda_{\mathrm{PL}}=524 \mathrm{~nm}\right), 89 \%$ $\left(\lambda_{\mathrm{PL}}=724 \mathrm{~nm}\right)$, and $35 \%\left(\lambda_{\mathrm{PL}}=415 \mathrm{~nm}\right)$, respectively, comparable to, or even higher than, those reported in the literature for the directly synthesized perovskite NCs. ${ }^{35,36,44}$ For pure $\mathrm{MAPbBr}_{3}$ and fully exchanged $\mathrm{MAPb}(\mathrm{Br} / \mathrm{I})_{3} \mathrm{NCs}$, the $\eta_{\mathrm{PL}}$ can reach high values (Fig. 3a). Similar behavior to PL characteristics upon halide mixing was also observed for all-inorganic $\mathrm{CsPbX}_{3} \mathrm{NCs}^{45}$ The drop of $\eta_{\mathrm{PL}}$ and its later recovery in the case of $\mathrm{MAPb}(\mathrm{Br} / \mathrm{I})_{3}$ mixtures can be attributed to halide segregation. ${ }^{46}$ The $\eta_{\mathrm{PL}}$ trend for $\mathrm{MAPb}(\mathrm{Br} / \mathrm{Cl})_{3}$ halide mixtures can also be associated with the ion migration processes. ${ }^{47}$ Both parent $\mathrm{MAPbBr}_{3}$ and mixed-halide $\mathrm{MAPb}(\mathrm{Br} / \mathrm{Cl})_{3}$ perovskite NCs $\left(\lambda_{\mathrm{PL}}=490-524 \mathrm{~nm}\right)$ show a plateau with a constantly high $\eta_{\mathrm{PL}}$ of approximately $85 \%$. For $\mathrm{MAPbBr}_{3-x} \mathrm{Cl}_{x}$ halide mixtures, when $x \leq 0.45$ (Br-rich), the ion migration in these mixed-halide perovskite materials seemed to be suppressed by $\mathrm{Cl}$ incorporation. This yielded an improvement of charge carrier transport in these materials. ${ }^{48}$ The suppression of ion migration within this $\mathrm{PL}$ emission range $\left(\lambda_{\mathrm{PL}}=490-524 \mathrm{~nm}\right)$ may explain the outstanding $\eta_{\mathrm{PL}}$. By further replacing $\mathrm{Br}$ with $\mathrm{Cl}$ in the mixedhalide $\mathrm{MAPb}(\mathrm{Br} / \mathrm{Cl})_{3}$, the $\eta_{\mathrm{PL}}$ decreases continuously, which can be attributed to an increasing population of halide vacancies and a boost of the ion migration within the crystal lattice. These defects introduce energy levels in the bandgap that may act as traps for photogenerated charge carriers. ${ }^{49}$ We also find that the optimized anti-solvent washing protocol could get rid of excess ligands in the $\mathrm{MAPb}(\mathrm{Br} / \mathrm{I})_{3}$ NCs with slight increase in $\eta_{\mathrm{PL}}$, for example, from $79 \%$ in unwashed $\mathrm{MAPb}(\mathrm{Br} / \mathrm{I})_{3}$ to $89 \%$ in the washed counterparts and further narrowing the emission bandwidth from $46 \mathrm{~nm}$ (unwashed) to $41 \mathrm{~nm}$ (washed) (ESI, $\dagger$ Fig. S13). Surprisingly, upon the anti-solvent washing treatment, the $\lambda_{\mathrm{PL}}$ of $\mathrm{MAPb}(\mathrm{Br} / \mathrm{I})_{3} \mathrm{NCs}$ also show a slight redshift from $721 \mathrm{~nm}$ to $724 \mathrm{~nm}$, whereas the optical density also decreases. Nevertheless, for the partially reacted $\mathrm{MAPb}(\mathrm{Br} / \mathrm{I})_{3}$ solutions, the anti-solvent washing would lead to a high degree of blueshift in the emission (ESI, $\dagger$ Fig. S14). ${ }^{50}$ To this end, in order to get rid of excess ligands for LEDs and prevent the PL emission from blueshifting during purification, partially anion-mixed $\mathrm{MAPb}(\mathrm{Br} / \mathrm{I})_{3}$ NCs were prepared by using the internanocrystal exchange method. On the other hand, for $\mathrm{MAPb}(\mathrm{Br} / \mathrm{Cl})_{3}$ samples, they are very robust upon anti-solvent washing. We observed a sudden drop of $\eta_{\mathrm{PL}}$ down to $11 \%$ in the yellowemitting $\mathrm{MAPb}(\mathrm{Br} / \mathrm{I})_{3}$ NCs. The low $\eta_{\mathrm{PL}}$ in the yellow-emitting $\operatorname{MAPb}(\mathrm{Br} / \mathrm{I})_{3}$ is attributed to the large population of non-radiative trap states possibly because of halide segregation ${ }^{46,51}$ that often emerged due to the fast dynamics of halide species within the crystal lattice. ${ }^{7}$ Our yellow-emitting $\mathrm{MAPb}(\mathrm{Br} / \mathrm{I})_{3}$ NCs are composed of a Br-rich environment within the crystal lattice as revealed by Energy-dispersive X-ray (EDX) spectroscopy (ESI, $\dagger$ Fig. S6). In particular, a number of reports have suggested that when 

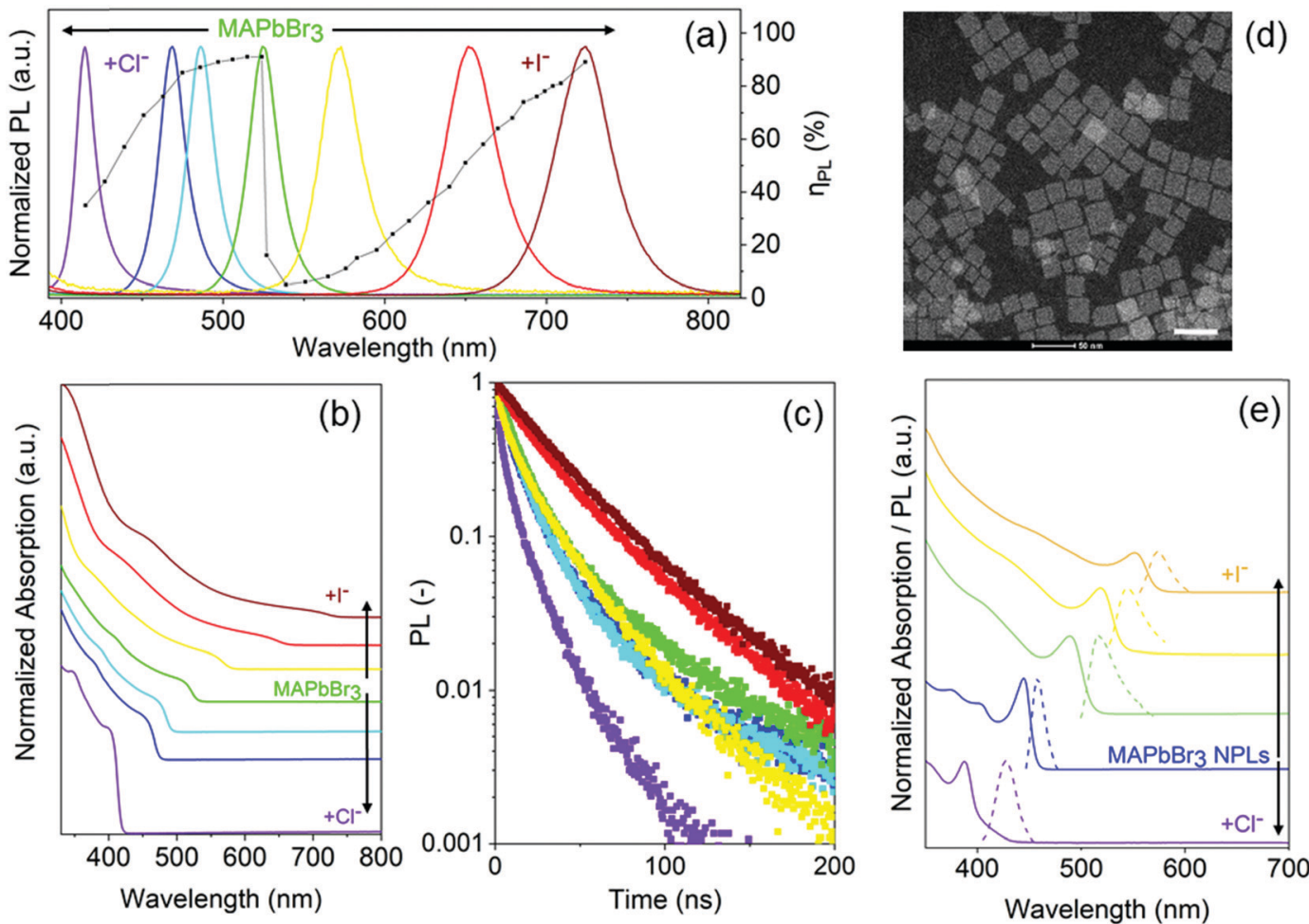

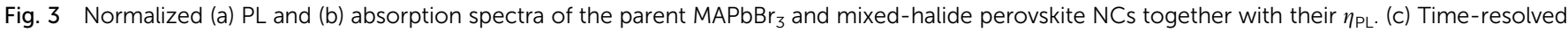
photoluminescence (TRPL) responses of the synthesized NCs. (d) Representative transmission electron micrograph of 2D MAPbBr $\mathrm{NPLs}_{3}$ (scale bar: $50 \mathrm{~nm}$ ). (e) Absorption (solid lines) and PL (dashed lines) of the LASPS synthesized mixed-halide perovskite NPLs.

$0.2<x<1$ in $\operatorname{MAPb}\left(\mathrm{Br}_{x} \mathrm{I}_{1-x}\right)_{3}$ (Br-rich), the halide segregation becomes more pronounced. ${ }^{46,52}$ Indeed, upon decreasing the $\mathrm{Br}$ content, the halide segregation seems suppressed as reflected by an increase in $\eta_{\mathrm{PL}}$. Recently, several trap passivation strategies have been exploited to mitigate halide segregation in perovskite materials such as $\mathrm{KI}, \mathrm{KBr}$, and iodide treatment. ${ }^{53-55}$

The colloidal stability for mixed-halide perovskite NCs is excellent, as reflected by the observation that high $\eta_{\mathrm{PL}}$ can retain over several months (ESI, $\uparrow$ Table S2). The outstanding colloidal stability of the parent $\mathrm{MAPbBr}_{3}$ and mixed-halides $\mathrm{MAPb}(\mathrm{Br} / \mathrm{Cl})_{3}$ and $\mathrm{MAPb}(\mathrm{Br} / \mathrm{I})_{3}$ could be attributed to longchain alkylamines such as oleylamine that regulate the kinetics of crystallization during the reactions. The oleic acids also prevent the NC aggregation, which results in the long-term colloidal stability of parent and exchanged halide NCs. ${ }^{15,56-58}$ We anticipate that the colloidal stability may be further improved by the post-synthetic treatment, which could passivate the trap-sites on the NC surfaces. ${ }^{59,60}$

The time-resolved photoluminescence (TRPL) responses of a number of representative samples are shown in Fig. 3c. The carrier lifetimes of $\mathrm{MAPbBr}_{3}$ and mixed-halide perovskite NCs are shown in ESI, $\uparrow$ Table S3. In general, we observed the average excited state lifetime, $\tau_{\text {avg, }}$, between 12.4 ns for fully reacted $\mathrm{MAPb}(\mathrm{Br} / \mathrm{Cl})_{3}$ to 40.4 ns for fully reacted $\mathrm{MAPb}(\mathrm{Br} / \mathrm{I})_{3}$ NCs. The yellow-emitting $\operatorname{MAPb}(\mathrm{Br} / \mathrm{I})_{3} \operatorname{NCs}\left(\lambda_{\mathrm{PL}}=573 \mathrm{~nm}\right)$ exhibit a relatively short $\tau_{\text {avg }}$ of $23.5 \mathrm{~ns}$ as compared to the parent green-emitting $\mathrm{MAPbBr}_{3}$ NCs, $\tau_{\text {avg }}=30.7$ ns. This is possibly due to its significantly low $\eta_{\mathrm{PL}}=11 \%$, as a result of the non-radiative trap sites caused by the halide segregation processes. $^{46}$

Finally, we demonstrated that the protocol also apply to the quantum-confined two-dimensional (2D) $\mathrm{MAPbBr}_{3}$ nanoplatelets (NPLs) (Fig. 3d), emitting at $455 \mathrm{~nm}$. Fig. 3e presents the absorption and PL spectra of parent blue-emitting $\mathrm{MAPbBr}_{3}$ NPLs and their halide exchanged products. In particular, the sharp excitonic absorption features are preserved upon halide mixing.

The protocols established here, including LASPS and the anti-solvent washing, allow us to prepare mixed-halide perovskite NCs for EL devices. The LED devices were fabricated using the parent $\mathrm{MAPbBr}_{3}$ and the anion exchanged $\mathrm{MAPb}(\mathrm{Br} / \mathrm{Cl})_{3}$ as well as $\mathrm{MAPb}(\mathrm{Br} / \mathrm{I})_{3}$ NCs. Fig. 4a schematically shows the device architecture with the layering sequence of ITO/PEDOT:PSS/ emissive layer/TPBi/LiF/Al. The energy diagram is shown in Fig. 4b. Five devices with EL emitting at $476 \mathrm{~nm}$ (blue), $524 \mathrm{~nm}$ (green), $584 \mathrm{~nm}$ (yellow), $656 \mathrm{~nm}$ (red), and $720 \pm 4 \mathrm{~nm}$ (nearinfrared, NIR) were fabricated (Fig. 4c). The EL emission bandwidths, the full width at half maximum (fwhm) range from 18 to $42 \mathrm{~nm}$ (Table 1). The Commission Internationale d'Eclairage (CIE) color coordinates for red, green, and blue devices yield a wide color gamut, covering $84 \%$ and $110 \%$ of the Rec. 2020 and NTSC standards in the $1931 \mathrm{CIE}$ color space (ESI, $\dagger$ Fig. S15). ${ }^{61}$ We were not able to obtain efficient deep-blue devices; otherwise the Rec. 2020 gamut coverage can reach $95 \%$, based on the measured $\lambda_{\mathrm{PL}}$ of our deep-blue-emitting $\mathrm{MAPb}(\mathrm{Br} / \mathrm{Cl})_{3}$ NCs.

The current density-voltage-luminance $(J-V-L)$ characteristics of our representative LEDs are shown in Fig. $4 \mathrm{~d}$ and e. The green 

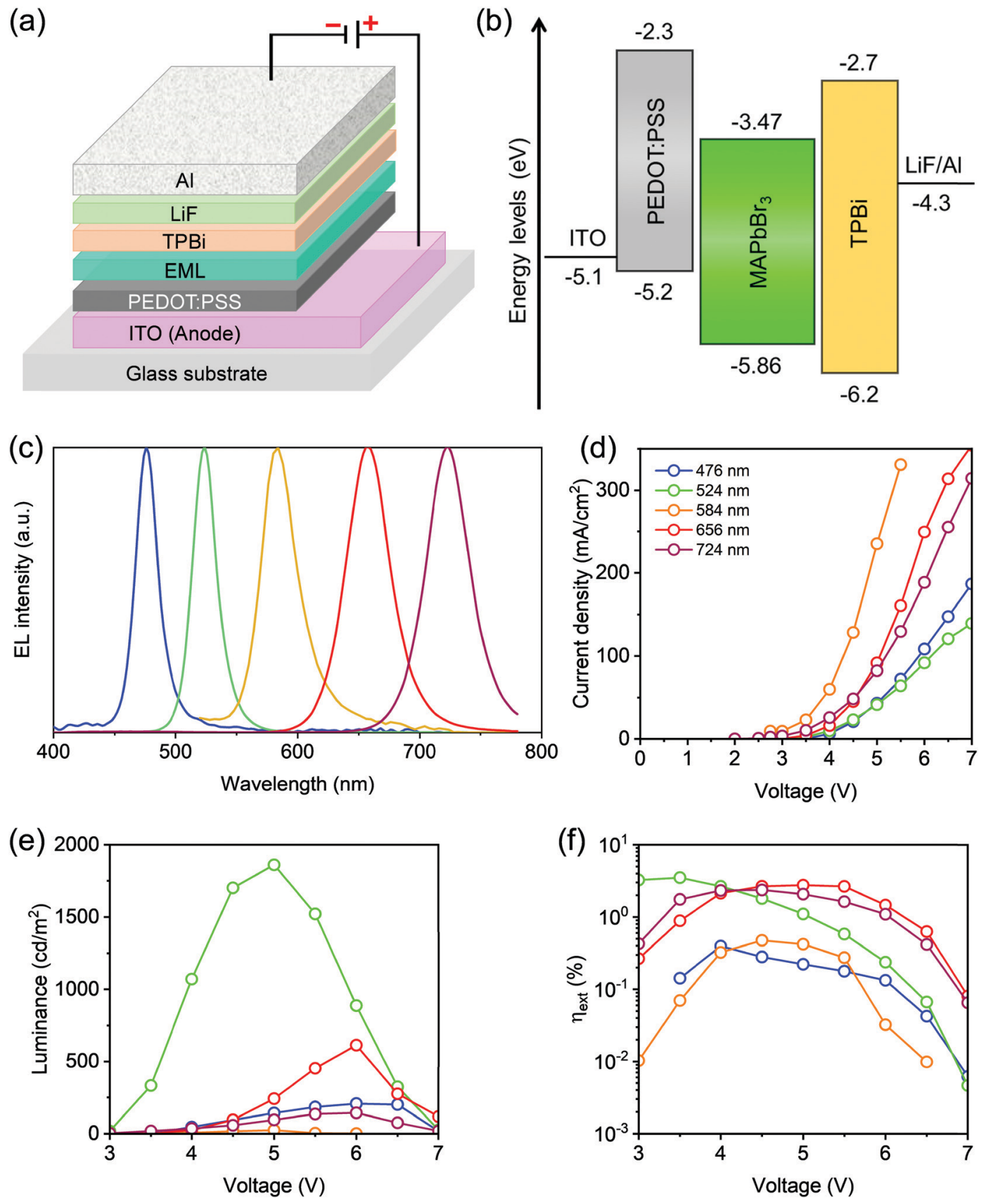

Fig. 4 EL characteristics using our synthesized anion exchanged MAPbX 3 perovskite NCs. (a) Schematic device architecture. (b) Schematic energy level diagram based on the parent $\mathrm{MAPbBr}_{3}$ NCs. (c) EL spectra of the fabricated LEDs. Characterized (d) current density, (e) luminance, and (f) external quantum efficiency as a function of voltage.

Table 1 EL characteristics for our fabricated LED devices using the parent $\mathrm{MAPbBr}_{3}$ (Green) and mixed-halide $\mathrm{MAPb}(\mathrm{Br} / \mathrm{Cl})_{3}$ and $\mathrm{MAPb}(\mathrm{Br} / \mathrm{l})_{3} \mathrm{NCs}$

\begin{tabular}{lllll}
\hline Device & $\eta_{\text {ext }}(\%)$ & $\lambda_{\text {EL }}(\mathrm{nm})$ & fwhm $(\mathrm{nm})$ & $\mathrm{CIE}_{x, y}$ coordinates \\
\hline Blue & 0.40 & 476 & 18.0 & $(0.125,0.144)$ \\
Green & 3.50 & 524 & 22.4 & $(0.150,0.771)$ \\
Yellow & 0.48 & 584 & 35.0 & $(0.476,0.391)$ \\
Red & 2.65 & 656 & 40.0 & $(0.705,0.290)$ \\
NIR & 2.36 & $720 \pm 4$ & 42.4 & $(0.644,0.320)$ \\
\hline
\end{tabular}

LED device based on parent $\mathrm{MAPbBr}_{3}$ NCs shows a turn-on voltage $\left(V_{\text {on }}\right)$ of $3 \mathrm{~V}$ with a luminance of $18.1 \mathrm{~cd} \mathrm{~m}^{-2}$. A maximum luminance of $1861 \mathrm{~cd} \mathrm{~m}^{-2}$ was realized at $5.0 \mathrm{~V}$ under a current density of $41.4 \mathrm{~mA} \mathrm{~cm} \mathrm{~cm}^{-2}$. The maximum external quantum efficiency, $\eta_{\text {ext }}$, reaches $3.5 \%$ (Fig. 4 f) corresponding to the maximum current efficiency, $\eta_{\mathrm{CE}}$, of $14.6 \mathrm{~cd} \mathrm{~A}^{-1}$. The blue and yellow devices reached relatively low $\eta_{\text {ext }}$ values of 0.40 and $0.48 \%$, respectively. The lower device performance of the blue LED device could be attributed to the deep lying valence band maximum (VBM) energy level, which is indeed the result of increasing the chloride content in the $\mathrm{MAPbX}_{3} \mathrm{NCs}^{9}$ that creates a high energy barrier for hole injection. ${ }^{62}$ It has been suggested that all forms of lead halide perovskites have a considerable 
(a)

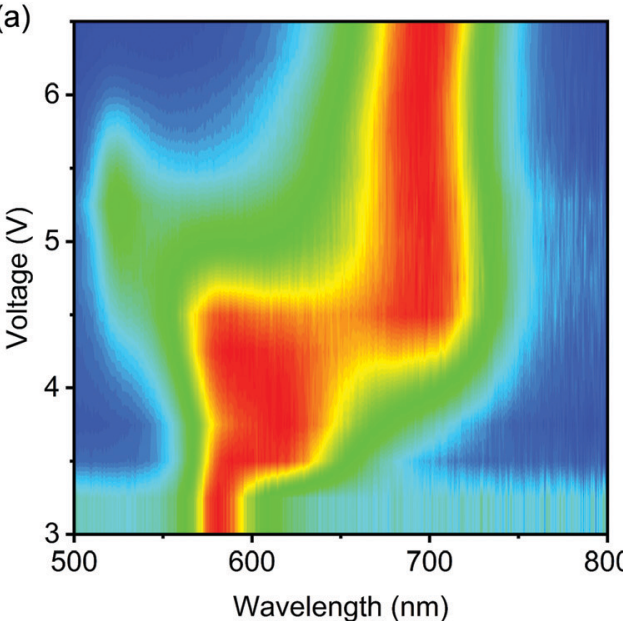

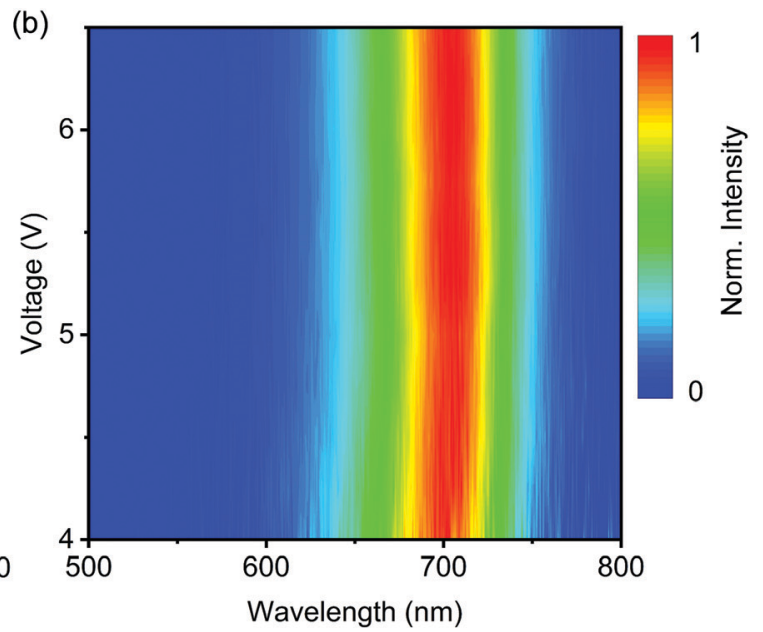

Fig. $5 \mathrm{EL}$ spectra of $\mathrm{KI}$-exchanged $\mathrm{MAPb}(\mathrm{Br} / \mathrm{l})_{3}$ under electrical stress. (a) The evolution of EL spectra as a function of driving voltage. (b) EL spectra of the color-stable mixed-halide $\mathrm{MAPb}(\mathrm{Br} / \mathrm{l})_{3} \mathrm{NCs}$ after applying a reverse bias of $-4 \mathrm{~V}$ for 20 minutes.

degree of electron traps; together with a high energy barrier for hole injection, the undesirable carrier imbalance would move the recombination zone closer to the hole transport layer (HTL)/ emissive layer interface. ${ }^{62,63}$

For $\operatorname{MAPb}(\mathrm{Br} / \mathrm{l})_{3} \mathrm{NCs}$, on the other hand, an expected low $\eta_{\text {ext }}$ and spectral stability of the yellow device is possibly due to its low $\eta_{\mathrm{PL}}$. Indeed, $\eta_{\mathrm{ext}}$ is substantially enhanced to $2.65 \%$ in the red device (Fig. 4f), following the increase of $\eta_{\mathrm{PL}}$ from 15 to $58 \%$. All EL spectra are consistent with their PL. It is noteworthy that our red-emitting device exhibits color coordinates of $(0.705,0.290)$, very close to those for the Rec. 2020 standard $(0.708,0.292){ }^{64}$ The NIR device reaches $\eta_{\text {ext }}$ of $2.36 \%$ at $720 \pm 4 \mathrm{~nm}$ with an fwhm of $42.4 \mathrm{~nm}$. The EL spectra of devices remained unchanged at different driving voltages (ESI, $\dagger$ Fig. S16). The LED devices based on parent $\mathrm{MAPbBr}_{3}$ NCs and mixed-halide perovskite $\mathrm{NCs}, \mathrm{MAPb}\left(\mathrm{Br} / \mathrm{l}_{3}\right.$ and $\mathrm{MAPb}(\mathrm{Br} / \mathrm{Cl})_{3}$, show excellent EL spectral stability over the entire operating voltage range. The ultimate EL spectral stability in the LEDs could be attributed to highly emissive NCs with a reduced number of defects. This could be explained by the synthesis via LASPS and the efficient purification protocols for the mixed-halide perovskite NCs. Advanced photophysical characterization is required to understand the origin of the observed spectral stability of LEDs. We also examined the operational stability of the resulting green, blue, and red LEDs based on $\mathrm{MAPbBr}_{3}, \mathrm{MAPb}(\mathrm{Br} / \mathrm{Cl})_{3}$, and $\mathrm{MAPb}(\mathrm{Br} / \mathrm{I})_{3} \mathrm{NCs}$, respectively. Our encapsulated devices showed the $\mathrm{LT}_{50}$, defined as the time of the luminance to decay to $50 \%$ of the initial luminance, of 23, 0.5, and 6.2 minutes, respectively, in the green, blue, and red LED devices (ESI, $\dagger$ Fig. S17). Note that all devices were stressed at a high constant $J=20 \mathrm{~mA} \mathrm{~cm}^{-2}$.

We found that potassium iodide (KI) powder can also be used as the solid source in the anion exchange reactions of $\mathrm{MAPbBr}_{3} \mathrm{NCs}$ (see ESI $\dagger$ for further details). In short, KI powder $\left(1.125 \mathrm{mmol}\right.$ ) was mixed with $\mathrm{MAPbBr}_{3}$ NCs for 2.5 hours, followed by centrifugation and MeOAc anti-solvent washing, yielding yellow-emitting solution $\left(\lambda_{\mathrm{PL}}=580 \mathrm{~nm}\right)$ with an $\eta_{\mathrm{PL}}$ of $10 \%$. The EL spectrum, however, tends to be unstable upon electrical excitation. Fig. 5a shows the evolution of the EL spectra as a function of driving voltage, which reveal the transformation from yellow to deep-red $(702 \mathrm{~nm})$ perovskite phase. It is clear that the deep-red-emitting phase that appears is not pure $\mathrm{MAPbI}_{3} \mathrm{NCs}$, but it is color-stable upon further electrical stress (Fig. 5b). The origin for the formation of this stable $\mathrm{MAPb}(\mathrm{Br} / \mathrm{I})_{3}$ phase is under further investigation. We anticipate that the electric-field-driven, irreversible post-synthetic technique may open a new avenue for color-stable perovskite LEDs.

In conclusion, we present a facile synthetic route, ligandassisted solid phase synthesis (LASPS), together with anti-solvent washing protocols for the preparation of electroluminescent colloidal NCs of mixed-halide lead perovskites. The synthesized $\mathrm{MAPb}(\mathrm{Br} / \mathrm{Cl})_{3}$ and $\mathrm{MAPb}(\mathrm{Br} / \mathrm{I})_{3} \mathrm{NCs}$ exhibit narrowband PL covering the entire visible spectral region. The protocols preserve the morphology, size, and shape of the perovskite NCs throughout the attainable halide composition. We also prepared quantumconfined mixed-halide perovskite NPLs, which have been difficult to synthesize using direct synthesis methods. Decent LED external quantum efficiencies were achieved using the standard device architecture. In particular, the red device reached color coordinates nearly identical to the newly defined Rec. 2020 standard. Further optimization of synthetic protocols, solid sources, and ligands may allow us to obtain high-efficiency and color-stable EL enduring electrical stress based on mixed-halide perovskite NCs.

\section{Conflicts of interest}

The authors declare that they have no other competing interests.

\section{Acknowledgements}

The authors are grateful for the financial support from the Swiss National Science Foundation (project number: 200021-178944), ETH research grant (ETH-33 18-2), and European Research Council (ERC) starting grant (N849229 - CQWLED). The authors 
are grateful to ScopeM (ETH Zürich) for access the electron microscopy facilities.

\section{References}

1 S. D. Stranks and H. J. Snaith, Nat. Nanotechnol., 2015, 10, 391.

2 A. Kojima, K. Teshima, Y. Shirai and T. Miyasaka, J. Am. Chem. Soc., 2009, 131, 6050-6051.

3 B. R. Sutherland and E. H. Sargent, Nat. Photonics, 2016, 10, 295-302.

4 Z.-K. Tan, R. S. Moghaddam, M. L. Lai, P. Docampo, R. Higler, F. Deschler, M. Price, A. Sadhanala, L. M. Pazos, D. Credgington, F. Hanusch, T. Bein, H. J. Snaith and R. H. Friend, Nat. Nanotechnol., 2014, 9, 687.

5 Y.-H. Kim, C. Wolf, Y.-T. Kim, H. Cho, W. Kwon, S. Do, A. Sadhanala, C. G. Park, S.-W. Rhee, S. H. Im, R. H. Friend and T.-W. Lee, ACS Nano, 2017, 11, 6586-6593.

6 S. F. Solari, S. Kumar, J. Jagielski and C.-J. Shih, J. Soc. Inf. Disp., 2019, 27, 667-678.

7 Q. A. Akkerman, G. Rainò, M. V. Kovalenko and L. Manna, Nat. Mater., 2018, 17, 394-405.

8 J. Jagielski, S. F. Solari, L. Jordan, D. Scullion, B. Blülle, Y.-T. Li, F. Krumeich, Y.-C. Chiu, B. Ruhstaller, E. J. G. Santos and C.-J. Shih, Nat. Commun., 2020, 11, 387.

9 J. Shamsi, A. S. Urban, M. Imran, L. De Trizio and L. Manna, Chem. Rev., 2019, 119, 3296-3348.

10 M. Hao, Y. Bai, S. Zeiske, L. Ren, J. Liu, Y. Yuan, N. Zarrabi, N. Cheng, M. Ghasemi, P. Chen, M. Lyu, D. He, J.-H. Yun, Y. Du, Y. Wang, S. Ding, A. Armin, P. Meredith, G. Liu, H.-M. Cheng and L. Wang, Nat. Energy, 2020, 5, 79-88.

11 W. van der Stam, J. J. Geuchies, T. Altantzis, K. H. W. van den Bos, J. D. Meeldijk, S. Van Aert, S. Bals, D. Vanmaekelbergh and C. de Mello Donega, J. Am. Chem. Soc., 2017, 139, 4087-4097.

12 B. Luo, F. Li, K. Xu, Y. Guo, Y. Liu, Z. Xia and J. Z. Zhang, J. Mater. Chem. C, 2019, 7, 2781-2808.

13 S. Kumar, J. Jagielski, S. Yakunin, P. Rice, Y.-C. Chiu, M. Wang, G. Nedelcu, Y. Kim, S. Lin, E. J. G. Santos, M. V. Kovalenko and C.-J. Shih, ACS Nano, 2016, 10, 9720-9729.

14 H. J. An, Y. C. Kim, D. H. Kim and J.-M. Myoung, ACS Appl. Mater. Interfaces, 2020, 12, 16726-16735.

15 S. Kumar, J. Jagielski, T. Marcato, S. F. Solari and C.-J. Shih, J. Phys. Chem. Lett., 2019, 10, 7560-7567.

16 L. Protesescu, S. Yakunin, M. I. Bodnarchuk, F. Krieg, R. Caputo, C. H. Hendon, R. X. Yang, A. Walsh and M. V. Kovalenko, Nano Lett., 2015, 15, 3692-3696.

17 G. Nedelcu, L. Protesescu, S. Yakunin, M. I. Bodnarchuk, M. J. Grotevent and M. V. Kovalenko, Nano Lett., 2015, 15, 5635-5640.

18 D. Zhang, Y. Yang, Y. Bekenstein, Y. Yu, N. A. Gibson, A. B. Wong, S. W. Eaton, N. Kornienko, Q. Kong, M. Lai, A. P. Alivisatos, S. R. Leone and P. Yang, J. Am. Chem. Soc., 2016, 138, 7236-7239.

19 N. Pellet, J. Teuscher, J. Maier and M. Grätzel, Chem. Mater., 2015, 27, 2181-2188.
20 T. Chiba, Y. Hayashi, H. Ebe, K. Hoshi, J. Sato, S. Sato, Y.-J. Pu, S. Ohisa and J. Kido, Nat. Photonics, 2018, 12, 681-687.

21 Q. A. Akkerman, V. D'Innocenzo, S. Accornero, A. Scarpellini, A. Petrozza, M. Prato and L. Manna, J. Am. Chem. Soc., 2015, 137, 10276-10281.

22 Y.-H. Suh, T. Kim, J. W. Choi, C.-L. Lee and J. Park, ACS Appl. Nano Mater., 2018, 1, 488-496.

23 B. A. Koscher, J. K. Swabeck, N. D. Bronstein and A. P. Alivisatos, J. Am. Chem. Soc., 2017, 139, 6566-6569.

24 N. Yarita, H. Tahara, M. Saruyama, T. Kawawaki, R. Sato, T. Teranishi and Y. Kanemitsu, J. Phys. Chem. Lett., 2017, 8, 6041-6047.

25 J. Pan, L. N. Quan, Y. Zhao, W. Peng, B. Murali, S. P. Sarmah, M. Yuan, L. Sinatra, N. M. Alyami, J. Liu, E. Yassitepe, Z. Yang, O. Voznyy, R. Comin, M. N. Hedhili, O. F. Mohammed, Z. H. Lu, D. H. Kim, E. H. Sargent and O. M. Bakr, Adv. Mater., 2016, 28, 8718-8725.

26 Y.-C. Chen, H.-L. Chou, J.-C. Lin, Y.-C. Lee, C.-W. Pao, J.-L. Chen, C.-C. Chang, R.-Y. Chi, T.-R. Kuo, C.-W. Lu and D.-Y. Wang, J. Phys. Chem. C, 2019, 123, 2353-2360.

27 X. Bao, M. Li, J. Zhao and Z. Xia, J. Mater. Chem. C, 2020, 8, 12302-12307.

28 M. V. Kovalenko, L. Protesescu and M. I. Bodnarchuk, Science, 2017, 358, 745.

29 D. Parobek, Y. Dong, T. Qiao, D. Rossi and D. H. Son, J. Am. Chem. Soc., 2017, 139, 4358-4361.

30 Y. J. Yoon, K. T. Lee, T. K. Lee, S. H. Kim, Y. S. Shin, B. Walker, S. Y. Park, J. Heo, J. Lee, S. K. Kwak, G.-H. Kim and J. Y. Kim, Joule, 2018, 2, 2105-2116.

31 C. Guhrenz, A. Benad, C. Ziegler, D. Haubold, N. Gaponik and A. Eychmüller, Chem. Mater., 2016, 28, 9033-9040.

32 Z. Yuan, Y. Shu, Y. Tian, Y. Xin and B. Ma, Chem. Commun., 2015, 51, 16385-16388.

33 J. De Roo, M. Ibáñez, P. Geiregat, G. Nedelcu, W. Walravens, J. Maes, J. C. Martins, I. Van Driessche, M. V. Kovalenko and Z. Hens, ACS Nano, 2016, 10, 2071-2081.

34 Y. Ida, Phys. Earth Planet. Inter., 1976, 13, 97-104.

35 Y. Hassan, O. J. Ashton, J. H. Park, G. Li, N. Sakai, B. Wenger, A.-A. Haghighirad, N. K. Noel, M. H. Song, B. R. Lee, R. H. Friend and H. J. Snaith, J. Am. Chem. Soc., 2019, 141, 1269-1279.

36 J. Shamsi, A. L. Abdelhady, S. Accornero, M. Arciniegas, L. Goldoni, A. R. S. Kandada, A. Petrozza and L. Manna, ACS Energy Lett., 2016, 1, 1042-1048.

37 T. Baikie, Y. Fang, J. M. Kadro, M. Schreyer, F. Wei, S. G. Mhaisalkar, M. Graetzel and T. J. White, J. Mater. Chem. A, 2013, 1, 5628-5641.

38 K.-H. Wang, L.-C. Li, M. Shellaiah and K. Wen Sun, Sci. Rep., 2017, 7, 13643.

39 Y. Liu, Z. Yang, D. Cui, X. Ren, J. Sun, X. Liu, J. Zhang, Q. Wei, H. Fan, F. Yu, X. Zhang, C. Zhao and S. Liu, Adv. Mater., 2015, 27, 5176-5183.

40 C. Quarti, E. Mosconi, J. M. Ball, V. D’Innocenzo, C. Tao, S. Pathak, H. J. Snaith, A. Petrozza and F. De Angelis, Energy Environ. Sci., 2016, 9, 155-163. 
41 P. P. Boix, S. Agarwala, T. M. Koh, N. Mathews and S. G. Mhaisalkar, J. Phys. Chem. Lett., 2015, 6, 898-907.

42 E. Edri, S. Kirmayer, M. Kulbak, G. Hodes and D. Cahen, J. Phys. Chem. Lett., 2014, 5, 429-433.

43 J. H. Noh, S. H. Im, J. H. Heo, T. N. Mandal and S. I. Seok, Nano Lett., 2013, 13, 1764-1769.

44 X. Liang, R. W. Baker, K. Wu, W. Deng, D. Ferdani, P. S. Kubiak, F. Marken, L. Torrente-Murciano and P. J. Cameron, React. Chem. Eng., 2018, 3, 640-644.

45 F. Krieg, S. T. Ochsenbein, S. Yakunin, S. ten Brinck, P. Aellen, A. Süess, B. Clerc, D. Guggisberg, O. Nazarenko, Y. Shynkarenko, S. Kumar, C.-J. Shih, I. Infante and M. V. Kovalenko, ACS Energy Lett., 2018, 3, 641-646.

46 A. J. Knight and L. M. Herz, Energy Environ. Sci., 2020, 13, 2024-2046.

47 T. Zhang, C. Hu and S. Yang, Small Methods, 2020, 4, 1900552. 48 N. Rybin, D. Ghosh, J. Tisdale, S. Shrestha, M. Yoho, D. Vo, J. Even, C. Katan, W. Nie, A. J. Neukirch and S. Tretiak, Chem. Mater., 2020, 32, 1854-1863.

49 D. Meggiolaro, E. Mosconi and F. De Angelis, ACS Energy Lett., 2019, 4, 779-785.

50 L. Yuan, R. Patterson, X. Wen, Z. Zhang, G. Conibeer and S. Huang, J. Colloid Interface Sci., 2017, 504, 586-592.

51 A. J. Knight, A. D. Wright, J. B. Patel, D. P. McMeekin, H. J. Snaith, M. B. Johnston and L. M. Herz, ACS Energy Lett., 2019, 4, 75-84.

52 Z. Xiao, L. Zhao, N. L. Tran, Y. L. Lin, S. H. Silver, R. A. Kerner, N. Yao, A. Kahn, G. D. Scholes and B. P. Rand, Nano Lett., 2017, 17, 6863-6869.

53 M. Abdi-Jalebi, Z. Andaji-Garmaroudi, S. Cacovich, C. Stavrakas, B. Philippe, J. M. Richter, M. Alsari, E. P. Booker, E. M. Hutter,
A. J. Pearson, S. Lilliu, T. J. Savenije, H. Rensmo, G. Divitini, C. Ducati, R. H. Friend and S. D. Stranks, Nature, 2018, 555, 497. 54 J.-N. Yang, Y. Song, J.-S. Yao, K.-H. Wang, J.-J. Wang, B.-S. Zhu, M.-M. Yao, S. U. Rahman, Y.-F. Lan, F.-J. Fan and H.-B. Yao, J. Am. Chem. Soc., 2020, 142, 2956-2967.

55 R. G. Balakrishna, S. M. Kobosko and P. V. Kamat, ACS Energy Lett., 2018, 3, 2267-2272.

56 F. Zhang, H. Zhong, C. Chen, X.-G. Wu, X. Hu, H. Huang, J. Han, B. Zou and Y. Dong, ACS Nano, 2015, 9, 4533-4542.

57 H. Huang, A. S. Susha, S. V. Kershaw, T. F. Hung and A. L. Rogach, Adv. Sci., 2015, 2, 1500194.

58 Y. Tong, F. Ehrat, W. Vanderlinden, C. Cardenas-Daw, J. K. Stolarczyk, L. Polavarapu and A. S. Urban, ACS Nano, 2016, 10, 10936-10944.

59 T. Chiba, Y. Takahashi, J. Sato, S. Ishikawa, H. Ebe, K. Tamura, S. Ohisa and J. Kido, ACS Appl. Mater. Interfaces, 2020, 12, 45574-45581.

60 Y. Liu, F. Li, Q. Liu and Z. Xia, Chem. Mater., 2018, 30, 6922-6929.

61 H.-W. Chen, R.-D. Zhu, J. He, W. Duan, W. Hu, Y.-Q. Lu, M.-C. Li, S.-L. Lee, Y.-J. Dong and S.-T. Wu, Light: Sci. Appl., 2017, 6, e17043.

62 J.-H. Jou, S. Kumar, A. Agrawal, T.-H. Li and S. Sahoo, J. Mater. Chem. C, 2015, 3, 2974-3002.

63 H. F. Haneef, A. M. Zeidell and O. D. Jurchescu, J. Mater. Chem. C, 2020, 8, 759-787.

64 H. Liang, F. Yuan, A. Johnston, C. Gao, H. Choubisa, Y. Gao, Y.-K. Wang, L. K. Sagar, B. Sun, P. Li, G. Bappi, B. Chen, J. Li, Y. Wang, Y. Dong, D. Ma, Y. Gao, Y. Liu, M. Yuan, M. I. Saidaminov, S. Hoogland, Z.-H. Lu and E. H. Sargent, Adv. Sci., 2020, 7, 1903213. 